\title{
Diamine oxidase plasma activities after treatment with heparin and jejunal morphometry in untreated coeliac disease
}

\author{
G R CORAZZA, L GINALDI, $\dagger$ A FALASCA, * A STROCCHI, C A ROSSI,* \\ D QUAGLINO, $\uparrow$ G GASBARRINI
}

From the Departments of I Medical Pathology and *Biochemistry, University of Bologna, and †Department of Internal Medicine, University of L'Aquila, Italy

SUMMARY Diamine oxidase plasma concentrations after treatment with heparin were measured and compared with the surface to volume ratio of jejunal biopsy samples assessed by a morphometric technique in patients with untreated and treated coeliac disease and in biopsied controls. As expected, enzyme activity was significantly lower in patients with untreated coeliac disease than in patients on a gluten-free diet and in biopsied controls. No difference was found betwen treated patients and biopsied controls. There was a significant overall correlation between plasma enzyme activity and surface to volume ratio of jejunal mucosa, although two untreated patients without an overt malabsorption syndrome but with a very low surface to volume ratio had normal enzyme activity.

This study shows that in coeliac disease plasma diamine oxidase activity after treatment with $\underset{\mathbb{\Phi}}{-}$ heparin does not always mirror the extent of the jejunal lesions, particularly in those patients with $\overrightarrow{0}$ minimal or unrelated symptoms who would benefit most from a valid screening test to identify the condition.

Diamine oxidase is an enzyme active in the mature upper villous cells of small bowel mucosa' and found in minute quantities in all other tissues, ${ }^{2}$ except the placenta. ${ }^{3}$ After treatment with heparin diamine oxidase is released into the plasma from the intestine, ${ }^{4}$ and it was thought that measurement of diamine oxidase plasma activity after treatment with heparin could be a peripheral marker of the maturity and integrity of the small intestine epithelium. In effect, it has been shown that this measurement is a sensitive test for estimating the length of acute intestinal mucosal injury in rats'; and decreased plasma concentrations after heparin treatment have been found in patients with untreated coeliac disease ${ }^{67}$ a condition characterised by atrophy of the intestinal villi and thus by a loss of mature enterocyte mass.

In this study we attempted to verify whether plasma concentrations of diamine oxidase after heparin treatment can accurately predict the degree of jejunal damage in coeliac disease assessed by morphometry. ${ }^{8}$

Accepted for publication 12 June 1989

\section{Material and methods}

In 26 adult patients with coeliac disease the diagnosis was based on a jejunal biopsy specimen showing the histological features of villous atrophy and improvement after at least six months of a gluten-free diet. Fourteen patients were on a gluten diet (untreated) and 12 on a gluten-free diet (treated) at the time of the study. Seven biopsied subjects, with normal jejunal mucosa, also took part in this study as controls. Informed consent was obtained from each patient and the investigation was approved by the local ethical committee.

\section{MEASUREMENT OF PLASMA ACTIVITY AFTER} HEPARIN TREATMENT

On the basis of a previous study of ours ${ }^{7}$ diamine oxidase plasma activity was measured 10 minutes after intravenous administration of heparin 150 units $/ \mathbf{k g}$. For biochemical assay the method of Okuyama and Kobayashi was used, ${ }^{9}$ as modified by Kusche et al, ${ }^{10}$ which measures the $\left[{ }^{14} \mathrm{C}\right]-\Delta_{1}$-pyrroline formation from $\left[{ }^{14} \mathrm{C}\right]$ putrescine. The assay mixture contained, in a final volume of $0.75 \mathrm{ml}, 150 \mu \mathrm{mol}$ of sodium potassium phosphate buffer, $\mathrm{pH} 7.6,0.2 \mathrm{ml}$ of plasma, and 
$750 \mathrm{nmol}$ of $\left[1,4-{ }^{14} \mathrm{C}\right]$ putrescine (New England Nuclear Co, Boston, Massachusetts, USA; specific activity $104.6 \mathrm{mCi} / \mathrm{mmol}$ ) diluted with unlabelled putrescine (15 mM) Sigma Chemical Co, St Louis, Missouri, USA) as substrate. After incubation for two hours at $37^{\circ} \mathrm{C}, 0.2 \mathrm{ml}$ of $70 \%$ perchloric acid, $1 \mathrm{ml}$ of strong alkaline buffer (pH 12.2), and $10 \mathrm{ml}$ of a toluene based scintillation mixture (1 litre of toluene containing $3.5 \mathrm{~g}$ of 2,5-dipheniloxazole) were added. This mixture served to extract the labelled reaction products, $\left[{ }^{14} \mathrm{C}\right]-\Delta_{1}$-pyrroline, and its spontaneously formed polymers. After shaking, the tubes were cooled at $-20^{\circ} \mathrm{C}$. When the water phase was frozen the toluene phase was transferred to counting phials. Radioactivity was measured by a Packard Tri-Carb C 2425 liquid scintillation spectrometer. For each determination two test proofs and two corresponding enzyme blanks were carried out. Plasma activity was expressed as units per millilitre, where 1 unit equals $1 \mathrm{nmol}$ of putrescine deaminated per hour at $37^{\circ} \mathrm{C}$.

On the basis of our previous results, ${ }^{7} 3 \cdot 2$ units $/ \mathrm{ml}$ were regarded as the lower limit of the control range.

\section{MORPHOMETRIC ASSESSMENT OF JEJUNAL BIOPSY} SPECIMENS

All 33 jejunal biopsy specimens were considered suitable for study according to the criteria of Perera $e t$ $a l .{ }^{11}$ All the specimens were taken from the region of the ligament of Treitz using the multipurpose tube ${ }^{12}$ as part of a routine diagnostic procedure. This tube allows four samples to be taken simultaneously. A preliminary examination by dissecting microscopy showed that there were no appreciable variations in mucosal changes between the four samples taken from any single patient. The biopsy specimens were fixed in buffered formalin, processed by standard methods, and after paraffin wax embedding, cut at $4 \mu \mathrm{m}$ thickness and stained with haematoxylin and eosin. Morphometric measurement of the slides was performed blind according to the method of Dunnill and Whitehead ${ }^{13}$ using a Weibel graticule (Graticules Ltd, Tunbridge Wells, Kent, England) inserted in the microscope eyepiece. ${ }^{8} \mathrm{~A}$ record was made of the number of times the lines cut the mucosal surface (c) and the number of hits (h)-that is, end points of the lines falling on the tissue section between the covering epithelium and the muscularis mucosae. At a constant magnification $(\times 125)$ the length (l) of each line of the graticule was $1.7 \times 10^{-2} \mathrm{~cm}$. The ratio of $\mathrm{c} / \mathrm{lh}$ was then calculated and regarded as a measure of surface area to volume ratio $(\mathrm{S} / \mathrm{V})$.

On the basis of our previous results, ${ }^{14} 15.6$ was regarded as the upper limit of the atrophic range.

The results were analysed by the non-parametric Wilcoxon rank sum test for unpaired data and by Spearman's rank correlation coefficient.

\section{Results}

The table shows the results from the 33 subjects studied. Plasma values after heparin treatment were significantly lower in untreated patients than in patients on a gluten-free diet $(p<0.001)$ and biopsied controls $(p<0.005)$. No significant difference was found between treated patients and biopsied controls. At the heparin doses used, no episodes of bleeding were recorded in any of the subjects studied.

With regard to the morphometric analysis of jejunal biopsy specimens, $\mathrm{S} / \mathrm{V}$ was significantly lower in untreated patients than in patients on a gluten-free diet $(p<0.001)$ and biopsied controls $(p<0.001)$. In patients on a gluten-free diet $S / V$ was significantly lower than in biopsied controls $(\mathrm{p}<0.005)$. There was no overlap between untreated and treated patients with coeliac disease.

Plasma values after heparin treatment showed a significant positive correlation with $\mathrm{S} / \mathrm{V}$ (figure). Two

Table Diamine oxidase plasma activity and surface area to volume ratio $(S / V)$ of jejunal biopsy specimens from three groups of patients studied

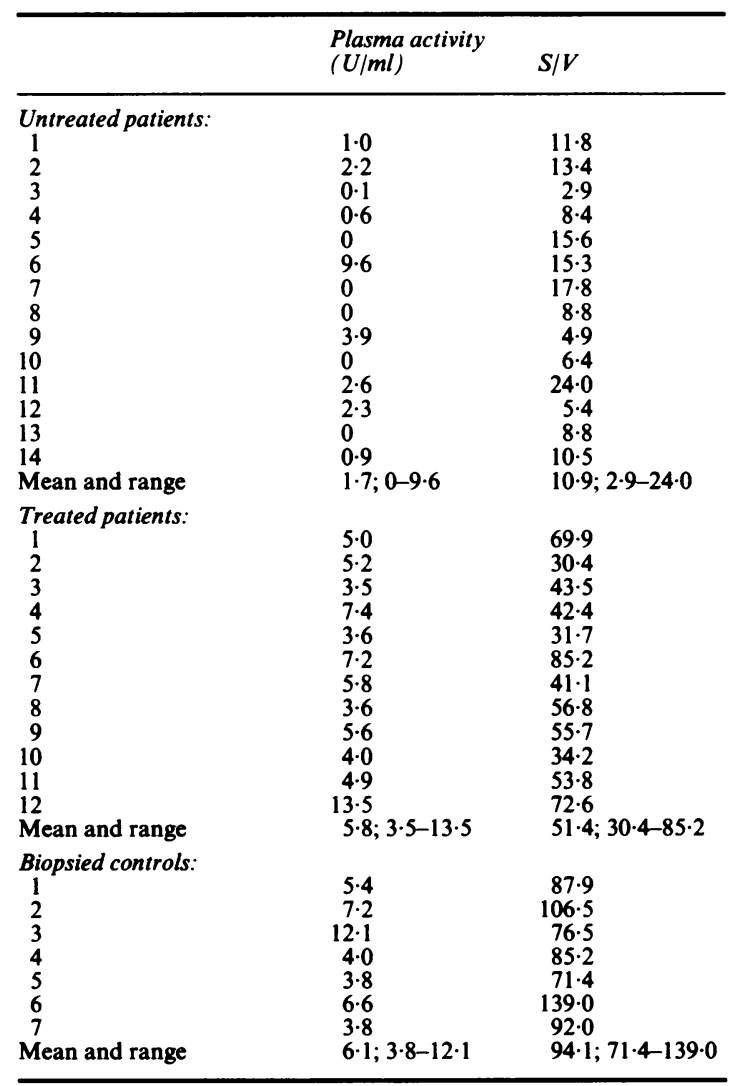




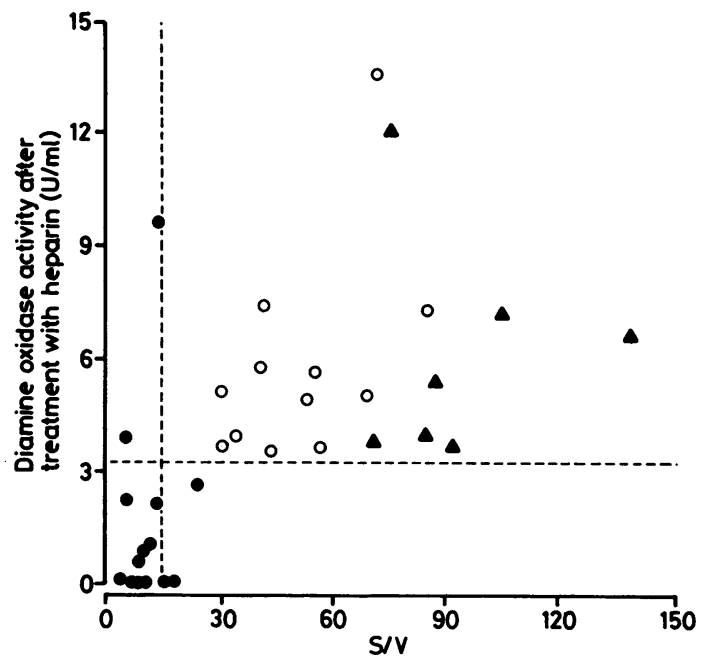

Figure Diamine oxidase plasma activities after heparin treatment plotted against surface to volume ratio $(S / V)$ in the three groups of patients studied (1) untreated patients, $\bigcirc$ treated patients, $\Delta$ biopsied controls). Dotted lines indicate the lower limit of the control range for plasma activities and the upper limit of the atrophic range for $S / V$.

untreated patients with an $\mathrm{S} / \mathrm{V}$ in the atrophic range, however, had plasma values in the normal range, while the $S / V$ in another two untreated patients with low plasma values was not in the atrophic range. In four out of 14 untreated patients, therefore, there were discrepancies between the two variables studied.

\section{Discussion}

In rats enterectomy counteracts the rise in circulating diamine oxidase activity after treatment with heparin, ${ }^{15}$ and in man a linear relation between intestinal mucosal diamine oxidase and plasma activities after heparin treatment has been shown, ${ }^{16}$ confirming that the small intestine is the major source of plasma diamine oxidase.

Intestinal diamine oxidase activity is synthesised by well differentiated and non-replicating enterocytes in the villous tip region. ${ }^{17}$ Because such cells are immature and reduced in number in coeliac disease, ${ }^{18}$ measurement after heparin treatment has mainly been used as a peripheral index of small bowel mucosal injury and recovery in this condition. Previous studies have, in fact, shown that plasma activity after heparin treatment is significantly decreased in untreated coeliac disease $^{67}$ and reverts to normal after gluten is removed from the diet. ${ }^{71}$ On the basis of these findings, the test was proposed to screen patients about to undergo intestinal biopsy and to follow up the response of patients with coeliac disease to a gluten-free diet, even though a correlation between plasma values after heparin treatment and the extent of jejunal lesions had never been ascertained.

The results of this study confirm that in untreated coeliac disease plasma diamine oxidase activity is reduced after heparin treatment, while after a glutenfree diet it does not differ from that in subjects whose intestinal mucosa was normal at morphometric assessment. In the quantitative assessment of jejunal biopsy specimens we used a morphometric technique which had previously proved highly reliable, ${ }^{14}$ and which in this study confirmed that there was a significant difference in the extent of jejunal lesions among the three groups studied We found a significant correlation between plasma diamine oxidase activities after heparin treatment and $S / V$, confirming that this enzyme is a non-invasive marker of jejunal damage (or integrity). Two untreated patients with low plasma values had an $S / V$ above but very close to the upper limit of the atrophic range and this indicates either that there were more severe lesions in other sites of the upper small intestine not explored by the biopsies, ${ }^{20}$ or that the test makes it possible to identify even minor lesions of the intestinal mucosa if they are extensive enough. In another two untreated patients with an S/V in the atrophic range, however, plasma values after heparin treatment fell within the normal range. Neither of these patients presented with clinical symp toms of overt malabsorption and both had a normap xylose test.

Coeliac disease is underdiagnosed, ${ }^{21}$ largely becaus many patients present with minimal, transient, of unrelated symptoms. ${ }^{22}$ It is obvious that a valid screening test should help to identify all those patients who are less easily suspected of having the disease on clinical grounds, but our results show that the test does not seem to have this property. In fact, although we showed a significant overall correlation between plasma values after heparin treatment and the extent of intestinal lesions, this association is not always present, especially in those with "trivial" presenting symptoms. Plasma values after heparin treatment, rather than the extent of jejunal lesions at a particular level, the region of the ligament of Treitz in our study, probably mirror the severity and extent of the lesions along the entire small bowel. ${ }^{2}$ Studies in animals have shown that diamine oxidase activity in the small bowel is three times greater in the ileum than in the jejunum. ${ }^{23}$ Thus in those cases of coeliac disease with proximal and limited lesions which result in more subtle and variable clinical expression, ${ }^{24}$ such as we found in two cases, the discrepancy between plasma diamine oxidase activity after heparin treatment and jejunal morphometry was not an unexpected finding. In patients with mucosal atrophy of the small bowel a significant inverse correlation between plasma values after heparin treatment and faecal fat excretion was 
shown, ${ }^{6}$ which contrasts with our results because it implies that the test can show even barely perceptible small bowel dysfunction. Unlike our study, however, D'Agostino et al referred in their study to patients with an overt malabsorption syndrome, most of whom had severe steatorrhoea, and did not include patients with mucosal damage and normal absorption. ${ }^{6}$

In treated coeliac disease, higher activities of diamine oxidase after heparin treatment mirror higher values of $\mathbf{S} / \mathbf{V}$ and this confirms that the test indirectly assesses the recovery of jejunal mucosa after a period on a gluten-free diet.

In conclusion, this study showed a significant overall correlation between plasma diamine oxidase activities after heparin treatment and the degree of jejunal damage assessed by morphometry in coeliac disease. In spite of this, plasma activity after heparin treatment does not always mirror the extent of jejunal lesions, particularly in those untreated patients who are less easily suspected of having the disease due to the absence of classic malabsorption symptoms.

\section{References}

1 Shakir KMM, Margolis S, Baylin SB. Localization of histaminase (diamine oxidase) in rat small intestinal mucosa: site of release by heparin. Biochem Pharmacol 1977;26:2343-7.

2 Luk GD, Bayless TM, Baylin SB. Diamine oxidase (histaminase). A circulating marker for rat intestinal mucosal maturation and integrity. J Clin Invest 1980;66:66-70.

3 Holinka CF, Gurpide E. Diamine oxidase activity in human decidua and endometrium. Am J Obstet Gynecol 1984;150:359 63.

4 Baylin SB, Beaven MA, Krauss RN, Keiser HR. Response of plasma histaminase activity to small doses of heparin in normal subjects and patients with hyperlipoproteinemia. J Clin Invest 1973;52:1985-93.

5 Luk GD, Bayless TM, Baylin SB. Plasma postheparin diamine oxidase. Sensitive provocative test for quantitating length of acute intestinal mucosal injury in the rat. $J$ Clin Invest 1983;71:1308-15.

6 D'Agostino L, Ciacci C, Daniele B, Barone MV, Sollazzo R, Mazzacca G. Postheparin plasma diamine oxidase in subjects with small bowel mucosal atrophy. Dig Dis Sci 1987;32:313-7.

7 Corazza GR, Falasca A, Strocchi A, Rossi CA, Gasbarrini G. Decreased plasma postheparin diamine oxidase levels in celiac disease. Dig Dis Sci 1988;33:956-61.

8 Corazza GR, Frazzoni M, Dixon MF, Gasbarrini G. Quantitative assessment of the mucosal architecture of jejunal biopsy specimens: a comparison between linear measurement, stereology, and computer aided microscopy. J Clin Pathol
1985;38:765-70.

9 Okuyama T, Kobayashi Y. Determination of diamine oxidase activity by liquid scintillation counting. Arch Biochem Biophys 1961;95:242-50.

10 Kusche J, Richter H, Hesterberg R, Schmidt J, Lorenz W. Comparison of the ${ }^{14} \mathrm{C}$-putrescine assay with the NADH test for the determination of diamine oxidase: description of a standard procedure with a high precision and an improved accuracy. Agents Actions 1973;3:148-56.

11 Perera DR, Weinstein WM, Rubin CE. Small intestinal biopsy. Hum Pathol 1975;6:157-217.

12 Brandborg LL, Rubin CE, Quinton WE. A multipurpose instrument for suction biopsy of the esophagus, stomach, small bowel and colon. Gastroenterology 1959;37:1-16.

13 Dunnill MS, Whitehead R. A method for the quantitation of small intestinal biopsy specimens. J Clin Pathol 1972;25:243-6.

14 Corazza GR, Bonvicini F, Frazzoni M, Gatto M, Gasbarrini G. Observer variation in assessment of jejunal biopsy specimens. A comparison between subjective criteria and morphometric measurement. Gastroenterology 1982;83:1217-22.

15 Rokkas T, Vaja S, Taylor P, Murphy GM, Dowling RH. Is the intestine the sole source of post-heparin plasma diamine oxidase (DAO)? Clin Sci 1988;75(suppl 19):1-51.

16 Rokkas T, Vaja S, Murphy GM, Dowling RH. Postheparin plasma diamine oxidase (DAO): a non-invasive marker of active ileal Crohn's disease? Gut 1986;27:A630.

17 Baylin SB, Steven SA, Shakir KMM. Association of diamine oxidase and ornithine decarboxylase with maturing cells in rapidly proliferating epithelium. Biochim Biophys Acta 1978;541:415-9.

18 Booth CC. Enterocyte in coeliac disease. I. Br Med J 1970;3 725-32.

19 D'Agostino L, Daniele B, Pignata S, et al. Postheparin plasma diamine oxidase increases in patients with coeliac disease during gluten free diet. Gut 1987;28(suppl 1):131-4.

20 Roy-Choudhury DC, Cooke WT, Banwell JG, Smits BJ. Multiple jejunal biopsies in adult coeliac disease. Am J Dig Dis 1967;12:657-63.

21 Swinson CM, Levi AJ. Is coeliac disease underdiagnosed? Br Med $J$ 1980;218:1258-60.

22 Logan RFA, Tucker G, Rifkind EA. Ferguson A. Changes in clinical features of coeliac disease in adults in Edinburgh and the Lothians 1960-79. Br Med J 1983;286:95-7.

23 Rokkas T, Vaja S, Taylor P, Murphy GM, Dowling RH. Enzymic regulation of mucosal polyamines in normal rat intestine. Gut 1988;29:A1478.

24 MacDonald WC, Brandborg LL, Flick AL, Trier JS, Rubin CE. Studies of celiac sprue. IV. The response of the whole length of the small bowel to a gluten-free diet. Gastroenterology 1964;47:573-89.

Requests for reprints to: G R Corazza, I Patologia Medica, Nuove Patologie, Policlinico S. Orsola, Via Massarenti 9, 40138 Bologna, Italy. 